\title{
Sobre la industria ósea de Altamira
}

\author{
Victoria Cabrera Valdés y Marta Gimenez la Rosa
}

Las últimas excavaciones en Altamira ofrecen cierta luz sobre el yacimiento y la probabilidad de interpretaciones que hasta el momento implicaban dificultades en cuanto a la atribución de industria. Con las dos referencias publicadas que ya existen (FREEMAN, L. G., 1988 y GONZALEZ ECHEGARAY, J., 1988) esta aportación se suma para completar en lo posible la visión del yacimiento desde el apartado de la industria ósea, con el estudio de la serie de piezas que se obtuvieron en 1980/81.

Antes de comenzar creemos conveniente resaltar algunos aspectos de aquellas campañas.

\section{LAS CARACTERISTICAS DE LA OCUPACIÓN}

Dos rasgos destacan de los resultados de esta campaña (FREEMAN, 1988). En primer lugar, las capas excavadas pertenecen unitariamente a una misma ocupación cultural, única y probablemente de corta duración, en la que se observaban diferenciaciones laterales y espaciales debidas a alteraciones sucesivas inferidas por actividades culturales. La secuencia, aunque corta, no estaba revuelta y era claramente diferenciada.

El material que aqui se estudia proviene del nivel 2, que contenía sumariamente varios rasgos: formación de conchero, combustión, pozos (B1, B2 y X) y «muretes", en los que se encontraban implícitas remociones realizadas por los mismos magdalenienses.

La fecha de C14 que se obtuvo (I-12012) $15.910 \pm 230$ B.P. concuerda claramente con la que se conocia anteriormente. 
Las capas magdalenienses están bien diferenciadas de las capas inferiores; si bien la base es irregular por lo que en una excavación antigua - sin métodos depurados de recuperación - la contaminación de piezas pudo ser un hecho factible en determinados puntos.

Todos estos hechos suponen una serie de factores importantes a la hora de observar la industria ósea.

La fragmentación antigua de la misma implica que las piezas que presentamos se debieron considerar probablemente desechos, vinculados en algunos casos a proyectiles recuperados con la carcasa del animal cazado, y en otros su fractura, quizá secundaria, viniera explicada por la alteración y remoción de los propios magdalenienses de sus estructuras anteriores. Sólo en un caso ha sido posible unir dos fragmentos de azagaya provenientes de dos cuadriculas diferentes, constituyéndose en un fragmento mayor y no en una pieza completa.

Por otro lado algunas piezas muestran un estado de conservación regular presentando áreas quemadas en su superficie, lo que está relacionado también con la reflexión anterior y los procesos de combustión inferidos.

Si bien la colección es numéricamente reducida, su fijación espacio/ temporal la hacen relevante. No cabe duda que al ser una serie con atribución clara y segura de nivel, asimismo bien datada, de un yacimiento del interés de Altamira, puede contribuir a aclarar y confirmar estudios e interpretaciones de las colecciones antiguas.

\section{ALGUNAS CONSIDERACIONES SOBRE EL ANÁLISIS DE LAS PIEZAS}

Ficha de estudio

La colección se ha estudiado aplicando un tratamiento informático que ha supuesto la confección de una ficha de trabajo que aporta toda la información posible sobre la pieza. Esta ficha se ha elaborado a partir de la utilizada por los investigadores que colaboran con el Centro de Investigación de Altamira, modificando aquellos aspectos convenientes a nuestro repertorio. 
La ficha se ha elaborado mediante el programa Professional File 1.0 (PFS), de Software Publishing Corporation, 1986. Esta ficha (o registro) consta de cuatro apartados subdivididos en una serie de campos, que son los que guardan la información.

El apartado primero es el IDENTIFICACIÓN de la pieza, que incluye los campos de sigla, item, nivel, fecha de excavación, cuadro, excavador, coordenadas y profundidad. Este apartado concluye con los campos familia, grupo, tipo primario y tipo secundario de la clasificación tipológica para industria ósea de I. Barandiarán (1967).

El segundo apartado es el de DESCRIPCIÓN, con los campos que especifican la materia prima de la pieza, las medidas, sección, el fuste y la conservación.

El tercer apartado se refiere a la DECORACIÓN, en sentido amplio, puesto que se incluyen todos los rasgos apreciables. En este caso, los campos se distribuyen en función de las caras de la pieza.

El último apartado se reserva para las OBSERVACIONES que vienen al caso, como por ejemplo posibles conexiones entre fragmentos, paralelos decorativos, etc.

\section{Sobre la identificación}

Conviene mencionar que las siglas de las piezas refieren la cuadricula, el nivel y el número de piezas. Aparecen dos piezas con la denominación de "revuelto" y que provienen de materiales recogidos al pie de la estratigrafía, cuya matriz era claramente reconocible del nivel 2. Máxime teniendo en cuenta que la capa solutrense no aparecia representada en el frente de la excavación, al iniciarse la misma. Se les denominó revuelto ya que su situación respecto a coordenadas y profundidad exacta era imposible determinarla.

Notas sobre la clasificación y atribución tipológica

La clasificación tipológica de la colección se ha realizado según la establecida por I. Barandiarán (1967). Los motivos por los que hemos escogido esta clasificación son la amplia aceptación que este sistema tiene en la investigación actual, su fácil comprensión y aplicación frente a otras de mayor complejidad. 
No obstante, hemos podido constatar algunos puntos en los que la sistematización elegida ha planteado algunas dudas en su aplicación.

- En algunos casos, la distinción entre varillas y azagayas presenta ciertas dificultades. Según la aceptada definición una varilla es un objeto de sección planoconvexa, rectangular o aplanada cuyo uso se estima por pares unidas por la cara ventral. Siguiendo este concepto hemos estimado las medidas de la sección como determinantes de su atribución a uno u otro grupo. De esta manera, sin obtener los indices de aplanamiento hemos llegado a la resolución que la separación superior a dos milimetros entre anchura y espesor ha determinado el considerarlas como secciones aplanadas correspondientes a varillas.

El criterio debiera unirse a los otros tres expuestos por I. Barandiarán en el estudio que realiza de los materiales de la cueva del Juyo (1987:184), pero ello era aún más dificil por las siguientes razones:

- "Dimensiones mayores... que nos llevarian a separar las de mayor entidad tipométrica (varilla) de las restantes (azagayas): control imposible de extremarse dada la extrema fragmentación de las evidencias". Dada la fragmentación, en el caso de la pequeña colección que nos ocupa es aún más perentorio. Asimismo la reconstrucción del total de la longitud de la pieza nos parece en extremo subjetivo dado el pequeño porcentaje de piezas que pudieran ser susceptibles de reconstrucción. A ello se suma una tendencia de las series cantábricas en las que la longitud de las piezas (completas) parece sensiblemente menor que las pertenecientes a otras series al norte de los Pirineos. Esta tendencia parece repetir la tendencia de las manifestaciones de la industria litica.

- "La presencia o no de series de estrias oblicuas sobre las caras internas o inferiores de las piezas: pues es más normal que las supuestas varillas posean esas caras 'planas' estriadas a todo lo largo...". En nuestro caso, las piezas con sección claramente cuadrangular tambien presentan esas finas estrias oblicuas que recorren el fuste sobre una cara que presenta los restos de tejido esponjoso interior del asta, lo cual no haría sino ampliar el número de varillas de la colección, muy amplio en consideración de otras colecciones y del criterio de la escasez de estas piezas.

Quizá fuera necesario ampliar el repertorio de azagayas por secciones aplanadas, para que pudiera ser más estricta la clasificación entre azagayas y varillas, teniendo siempre en cuenta que las medidas de longitud y anchura se han tomado en el sector que marcaba más claramente la zona mesial del fuste. 
En las azagayas de sección cuadrada, sobre un total de siete piezas, en tres casos se observan los mismos valores de anchura y grosor, en dos hay una diferencia de un milimetro y en otras dos la diferencia alcanza los dos milímetros, siempre tomando las medidas en el punto más próximo a lo que constituiria la parte mesial del fuste. Según este criterio se ha evidenciado el hecho de que tanto varillas (en cualquiera de sus tipos) como azagayas de sección cuadrada presentan la misma morfología, y que esos dos milímetros de diferencia, aún siendo arbitrarios, se han constituido como único criterio efectivo para incluir la pieza en uno u otro grupo.

- Enlazando con este concepto, también observamos que la clasificación de las piezas fragmentadas en extremos proximales o distales presentaba problemas cuando no habia elementos característicos (biseles cóncavos, bases redondeadas, etc.). Hemos tomado como criterios para los fragmentos distales los que portan decoración de trazos cortos perpendiculares al fuste (“marcas de caza») y los que muestran un aguzamiento significativo.

\section{Sobre las decoraciones}

En lo referente a los procesos de elaboración de la pieza hasta la fase final hemos tenido en cuenta tres pasos:

1. La fase primaria estaría representada por el desbastado y extracción de la forma general del artefacto.

2. La fase secundaria estaria relacionada con los procesos de acabado morfológico, en el que se incluirian las huellas de pulimento, abrasión, factura y trazos utilitarios.

En esta fase hay dos momentos bien diferenciados, uno inicial más relacionado con gestos tecnológicos (los referentes al aspecto morfológico: abrasión, pulimento, etc.) y un segundo momento, de carácter final, con trazos funcionales o utilitarios que evidencian el acabado de la pieza, por ejemplo los destinados a asegurar enmangues y la unión de las caras ventrales de varillas y azagayas de sección cuadrada o los posiblemente dedicados a portar sustancias tóxicas.

3. La tase terciaria vendría dada por la incidencia de gestos no relacionados con ninguna función técnica o morfológica, y que estarian vinculados a una representación probablemente de carácter social o cultural, no directamente económica: la decoración. 
Hemos dejado fuera de estas fases los trazos que se han producido por el uso de las piezas, en una fase posterior a la terciaria, aspecto este que no afecta a los criterios de carácter cultural que nos ocupan.

El estudio de los trazos que se engloban tanto en la fase secundaria como en la primaria se ha realizado teniendo en cuenta su desarrollo en las cuatro caras posibles de la pieza (dorsal, lateral izquierdo, ventral y lateral derecho).

Desglose de la colección

Los materiales que entran en la clasificación analítica de I. Barandiarán constituyen un total de 38 piezas, desglosadas básicamente en la familia de "apuntados" (el 86,61 por ciento de la colección), y minoritariamente en la familia de "perforados" (el 12,82 por ciento de la colección).

La distribución, expresada en tantos por ciento, por grupos tipológicos se resume en la tabla siguiente:

TABLA 1

\begin{tabular}{lrr}
\hline GRUPO & $\begin{array}{c}\text { TOTAL } \\
\text { PIEZAS }\end{array}$ \\
\hline I & 16 & $\%$ \\
VI & 2 & 43,37 \\
VIII & 15 & 5,27 \\
IX & 1 & 36,95 \\
& 4,76 \\
\hline
\end{tabular}

Los grupos representativos lo constituyen el I y el VIII (puntas o azagayas y varillas), siendo minoritarios el XXI (agujas, con cuatro ejemplares) y el VI (2 piezas apuntadas). Presenta un solo ejemplar el grupo IX, una espátula.

El listado general de las piezas se ofrece en la tabla $n .^{\circ} 2$, en el que se pueden seguir cinco campos: identificación, grupo, tipo 1 , tipo 2 y sección de la pieza. La fracturación del material impide en muchos casos establecer la clasificación de tipos primarios y aún más si cabe en tipos secundarios. 
TABLA 2

\begin{tabular}{|c|c|c|c|c|}
\hline SIGLA & GRUPO & TIPO 1 & TIPO 2 & SECC. \\
\hline K8.2.10 & VIII & & & rectangular \\
\hline K8.2.11 & VIII & 29 & 2 & rectangular \\
\hline K8.2.2 & 1 & 1 & 5 & elíptica \\
\hline K8.2.3 & $X X I$ & & & circular \\
\hline K8.2.5 & VI & 18 & 1 & circular \\
\hline K8.2.6 & VIII & & & rectangular \\
\hline K8.2.7 & VIII & & & rectangular \\
\hline K8.2.8 & VI & 18 & & pl. cvxa \\
\hline K8.4 & 1 & & & circular \\
\hline K9.2.1 & 1 & & & circular \\
\hline K9.2.10 & VIII & 27 & 2 & rectangular \\
\hline K9.2.11 & I & & & cuadrada \\
\hline K9.2.12 & $X X 1$ & 55 & & circular \\
\hline K9.2.13 & VIII & & & rectangular \\
\hline K9.2.2 & 1 & & & circular \\
\hline K9.2.3 & 1 & & & circular \\
\hline K9.2.4 & VIII & 27 & 2 & rectangular \\
\hline K9.2.5 & 1 & 4 & 6 & cuadrada \\
\hline K9.2.6 & $X X I$ & 55 & 1 & circular \\
\hline K9.2.7 & $X X I$ & 55 & & circular \\
\hline K9.2.8 & 1 & & & circular \\
\hline K9.2.9 & 1 & & & cuadrada \\
\hline L9.2.1 & IX & 31 & & aplanada \\
\hline L9.2.10 & VIII & 29 & 2 & rectangular \\
\hline L9.2.11 & VIII & 27 & 2 & rectangular \\
\hline L9.2.12 & 1 & & & cuadrada \\
\hline L9.2.14 & 1 & & & cuadrada \\
\hline L9.2.15 & VIII & & & rectangular \\
\hline L9.2.16 & VIII & 26 & 1 & pl. cvxa \\
\hline L9.2.2 & VIII & & & rectangular \\
\hline L9.2.4 & VIII & 26 & 2 & rectangular \\
\hline L9.2.5 & 1 & & & circular \\
\hline L9.2.6 & 1 & & & cuadrada \\
\hline L9.2.7 & 1 & 4 & 4 & circular \\
\hline L9.2.8 & 1 & & & cuadrada \\
\hline M9.2.1 & VIII & & & rectangular \\
\hline revuelto 2 & 1 & & & circular \\
\hline revuelto 3 & VIII & & & rectangular \\
\hline
\end{tabular}


DESGLOSE DE LOS GRUPOS TIPOLÓGICOS MÁS SIGNIFICATIVOS

\section{Grupo 1: Puntas o Azagayas}

Este es el grupo que muestra el mayor número de piezas, hechas sobre asta, del repertorio: un total de dieciséis. Sólo se pueden identificar los tipos primario y secundario de tres piezas, ya que el resto son fragmentos que no permiten su correcta clasificación (tabla n. ${ }^{\circ} 3$ ).

Las secciones que presenta el grupo I se distribuyen en un 50 por ciento de circulares, un 43,8 por ciento de cuadrangulares y un 6,25 por ciento (un pieza: K8.2.2) que tiene la sección eliptica. Este término le hemos considerado incluir, dada la reciente descripción de algunos de estos objetos (Delporte et al. 1988), ya que sus dimensiones de anchura y grosor son 11 y $8 \mathrm{~mm}$.

Grupo VIII: Varillas

De un total de quince piezas, catorce $(93,33$ por ciento) tienen la sección rectangular y una $(6,66$ por ciento) es de sección planoconvexa. Para establecer esta clasificación nos hemos atenido a los tipos primarios $26,27,28$ y 29 ya que son los que definen la morfología de la pieza, considerando que la decoración es un atributo terciario. Este grupo presenta un número elevado de piezas en las que se ha podido establecer sus tipos primario y secundario. En cuanto a sus bases dos piezas las tienen redondeada o apuntada, tres tienen monobisel oblícuo y dos tienen la base biselada (Tabla 3).

Al igual que las piezas del grupo I, las del grupo VIII presentan como materia prima asta.

\section{Otros grupos}

Representados por un perforador y un fragmento más (Grupo VI), un fragmento distal de espátula (Grupo IX) y cuatro fragmentos de agujas (Grupo XXI), todos ellos mesiales y de pequeño tamaño, sin rasgos decorativos. 
TABLA 3

\begin{tabular}{|c|c|c|c|c|c|c|}
\hline GRUPO & TIPO. 1 & TIPO.2 & A & $G$ & SECC & SIGLA \\
\hline 1 & & & 10 & 8 & cuadrada & L9.2.14 \\
\hline 1 & & & 10 & 9 & circular & revuelto 2 \\
\hline 1 & & & 11 & 9 & cuadrada & L9.2.12 \\
\hline 1 & & & 3 & 3 & circular & K9.2.2 \\
\hline 1 & & & 5 & 5 & circular & $K 8.4$ \\
\hline 1 & & & 5 & 5 & circular & K9.2.1 \\
\hline 1 & & & 5 & 5 & circular & $\mathrm{K} 9.2 .8$ \\
\hline 1 & & & 6 & 5 & cuadrada & $\mathrm{k} 9.2 .9$ \\
\hline 1 & & & 6 & 5,5 & circular & L9.2.5 \\
\hline 1 & & & 7 & 5 & circular & K9.2.3 \\
\hline 1 & & & 7 & 5 & cuadrada & L9.2.8 \\
\hline 1 & & & 7 & 7 & cuadrada & L9.2.6 \\
\hline 1 & & & 8 & 8 & cuadrada & K9.2.11 \\
\hline 1 & 1 & 5 & 11 & 8 & eliptica & K8.2.2 \\
\hline 1 & 4 & 4 & 5 & 5 & circular & ᄂ9.2.7 \\
\hline 1 & 4 & 6 & 4 & 4 & cuadrada & K9.2.5 \\
\hline VIII & & & 10 & 7 & rectangular & $K 8.2 .10$ \\
\hline VIII & & & 12 & 6.5 & rectangular & L9.2.15 \\
\hline VIII & & & 12 & 8 & rectangular & L9.2.2 \\
\hline Vill & & & 12 & 9 & rectangular & K9.2.13 \\
\hline VIII & & & 12,5 & 8,5 & rectangular & M9.2.1 \\
\hline VIII & & & 9 & 6 & rectangular & $K 8.2 .7$ \\
\hline VIII & & & 9 & 6 & rectangular & revuelto 3 \\
\hline VIII & & & 9 & 6,5 & rectangular & K8.2.6 \\
\hline VIII & 26 & 1 & 10 & 6 & pl. cvxa & L9.2.16 \\
\hline VIII & 26 & 2 & 9 & 6,5 & rectangular & L9.2.4 \\
\hline VIII & 27 & 2 & 10 & 8 & rectangular & L9.2.11 \\
\hline VIII & 27 & 2 & 11 & 8 & rectangular & K9.2.4 \\
\hline VIII & 27 & 2 & 12 & 9 & rectangular & K9.2.10 \\
\hline VIII & 29 & 2 & 12 & 8 & rectangular & L9.2.10 \\
\hline VIII & 29 & 2 & 8 & 6 & rectangular & K8.2.11 \\
\hline
\end{tabular}




\section{ESTUDIO DE LA DECORACIÓN}

Para este estudio se han tenido en cuenta las piezas englobadas en los grupos tipológicos I y VIII.

Una primera clasificación nos ha llevado a establecer una serie de categorías:

a) Piezas en las que sólo se observan trazos de la fase terciaria. Hay tres sobre un total de 35 , lo que supone un 8,6 por ciento de la colección

b) Piezas que muestran conjuntamente trazos de la fase terciaria y secundaria tanto de su momento final como inicial. Representan un 37,2 por ciento con 13 piezas.

c) Piezas que muestran trazos sólo de la fase secundaria en sus dos momentos, son cinco piezas que suponen un 14,2 por ciento.

d) Piezas sin trazos apreciables. catorce, que representan el 40 por ciento del total del repertorio.

En la colección que presentamos todos los trazos decorativos que aparecen en las piezas son los considerados comúnmente como signos, sin que en ningún caso se encuentren motivos figurativos (Tabla 4).

Hemos observado la existencia de signos simples y complejos, atendiendo a criterios ya establecidos en los estudios de arte mueble.

Una primera evaluación nos ha llevado a observar la relación entre los motivos utilizados y su asociación con las caras de las piezas. De esta observación se obtienen las siguientes conclusiones:

\section{TRAZOS DECORATIVOS COMPLEJOS}

Hemos podido constatar dos tipos de trazos complejos, uno ya conocido como "tectiforme Altamira" (UTRILLA, 1981:287) y motivos en forma de aspa.

El "tectiforme Altamira" aparece en tres ocasiones, siempre sobre la cara dorsal de las piezas. En solo una pieza aparece aislado (K8.2.10). En un caso (L9.2.14) está junto a trazos funcionales de factura y en dos casos con otros trazos en las caras laterales más funcionales oblícuos 
TABLA 4

\begin{tabular}{|c|c|}
\hline$K 8.2 .10$ & fto. distal de varilla \\
\hline K8.2.11 & fto proximal de varilla \\
\hline $\mathrm{K} 8.2 .2$ & fto. distal de azagaya \\
\hline $\mathrm{K} 8.2 .3$ & fo. mesial de aguja \\
\hline K8.2.5 & punzón \\
\hline K8.2.6 & fto. mesial de varilla \\
\hline $\mathrm{K} 8.2 .7$ & fto. mesial de varilla \\
\hline$K 8.2 .8$ & fto. de punzón \\
\hline K8.4 & fto distal de azagaya \\
\hline K9.2.1 & fto. mesial de azagaya \\
\hline $\mathrm{K} 9.2 .10$ & tho proximal de varilla \\
\hline $\mathrm{K} 9.2 .11$ & fto. mesial de azagaya \\
\hline K9.2.12 & fto. mesial de aguja \\
\hline K9.2.13 & fto. distal de varilla \\
\hline $\mathrm{K} 9.2 .2$ & fto distal de azagaya \\
\hline $\mathrm{K} 9.2 .3$ & fto distal de azagaya \\
\hline K9.2.4 & Ho distal de varilla \\
\hline K9.2.5 & azagaya completa \\
\hline $\mathrm{k} 9.2 .6$ & fto. distal de aguja \\
\hline $\mathrm{k} 9.2 .7$ & fto mesial de aguja \\
\hline K9.2.8 & fto. distal de azagaya \\
\hline $\mathrm{K} 9.2 .9$ & fto mesial de azagaya \\
\hline$\llcorner 9.2 .1$ & fto distal de espatula \\
\hline L9.2.10 & to proximal de varilla \\
\hline L9.2.11 & fto proximal de varilla \\
\hline L9.2.12 & to. distal de azagaya \\
\hline L9.2.14 & to mesial de azagaya \\
\hline L9.2.15 & tto distal de varilla \\
\hline ᄂ9.2.16 & fto mesial de varilla \\
\hline$\llcorner 9.2 .2$ & Ho. distal de varilla \\
\hline L9.2.4 & to. distal de varilla \\
\hline L9.2.5 & tto distal de azagaya \\
\hline L9.2.6 & fto. proximal de azagaya \\
\hline L9.2.7 & azagaya completa \\
\hline L9. 2.8 & tho. mesial de azagaya \\
\hline M9.2.1 & fto de varilla \\
\hline revuelto 2 & fto. distal de azagaya \\
\hline revuelto 3 & Ho. mesial de varilla \\
\hline
\end{tabular}

decorativa
funcional y decorativa
funcional
sin decoración
funcional
funcional
sin decoración
sin decoración
decorativa
sin decoración
funcional y decorativa
funcional y decorativa
funcionales
funcional y decorativa
sin decoración
sin decoración
funcional y decorativa
funcional y decorativa
sin decoración
sin decoración
sin decoración
funcional y decorativa
huellas de uso
funcional y decorativa
funcional y decorativa
funcional
funcional y decorativa
funcional
funcional y decorativa
sin decoración
funcional y decorativa
sin decoración
sin decoración
sin decoración
decorativa
funcional
sin decoración
funcional y decorativa

decorativa

funcional

sin decoración

funcional

funcional

decorativa

funcional y decorativa

funcionales

funcional y decorativa

sin decoracion

funcional y decorativa

funcional y decorativa

sin decoración

funcional y decorativa

huellas de uso

funcional y

funciona

funcional y decorativa

funciona

funcional y decorativa sin decoración

funcional y decorativa

in decoración

decorativa

sin decoración

funcional y decorativa 

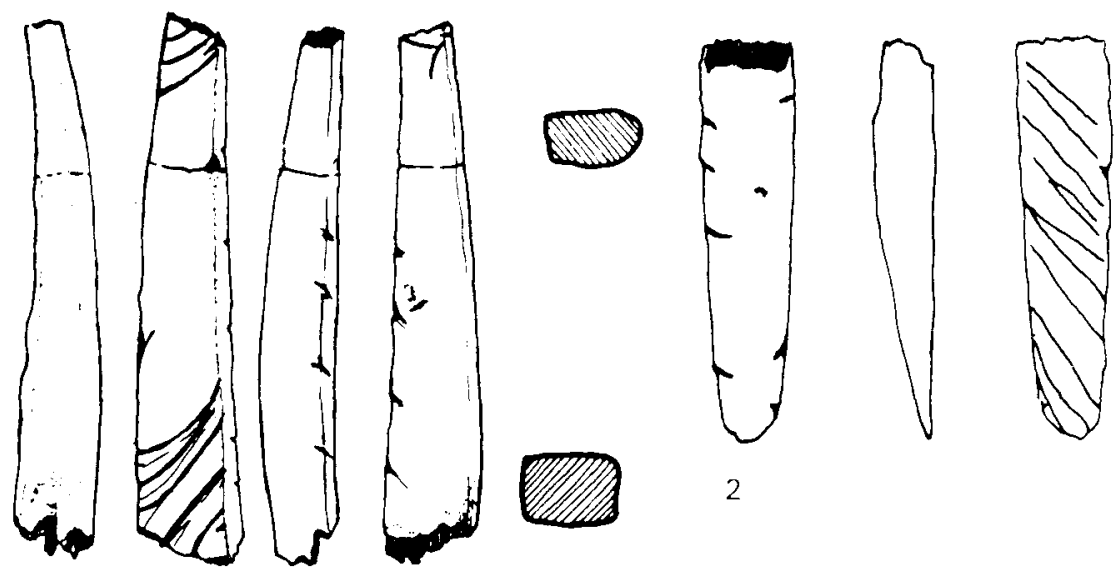

2

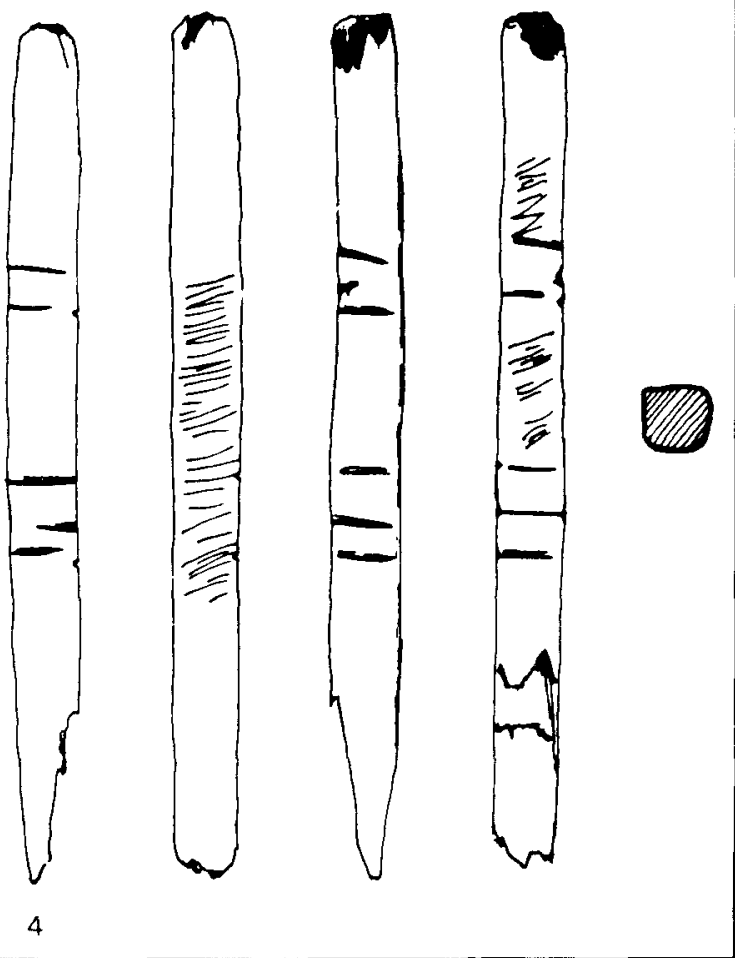

Fig. 1. Industria ósea de Altamira 1981. 1) K9.2.13; 2) $(9.2 .11$; 3) K8.2.2 y 4) K9.2.11. 
en la cara ventral (L9.2.14 y L9.2.10). Este motivo se encuentra sobre dos varillas (K8.2.10 y L9.2.10). y sobre una azagaya (L9.2.14).

En ningun caso se produce la asociación de trazos complejos de la fase terciaria (decorativos) con cara ventral de la pieza. Solo una pieza (revuelto 3) muestra trazos simples en esta posición. El resto de piezas presentan trazos funcionales o ausencia de trazos en esta cara ventral.

En las caras laterales los motivos complejos que aparecen son aspas, en una misma pieza; con la particularidad de que en el lateral derecho se presenta de forma singular, mientras que en lateral izquierdo aparece repetida. Esta pieza, una azagaya de sección cuadrada (K9.2.9) muestra en su cara dorsal la superficie natural del asta, sin que haya evidencias de una intención de pulirla o eliminarla.

\section{TRAZOS DECORATIVOS SIMPLES}

Constituyen los elementos más abundantes de este repertorio de piezas. Para su estudio hemos establecido unos criterios atendiendo en primer lugar a su disposición respecto al eje de la pieza; de este modo hemos obtenido trazos longitudinales, oblicuos y perpendiculares.

A continuación se ha tenido en cuenta la incidencia del trazo sobre la superficie de la pieza, de modo que su división se establece en profundos, superficiales, anchos y finos.

Respecto a la forma de los trazos hay dos grandes grupos, rectos y curvados. Por último, hemos observado la asociación de los trazos respecto a si mismos, de manera que existen trazos agrupados en haces y trazos aislados formando conjuntos.

Los trazos curvados sólo parecen en un fragmento mesial de azagaya de sección cuadrada (L9.2.8), es decir, son los menos representados. Se encuentran en las caras dorsal y lateral derecha y en ambos casos son longitudinales profundos.

Sólo en una ocasión se sitúan trazos simples oblicuos no funcionales en la cara ventral de las piezas (revuelto 3 ). Se ha evidenciado que los trazos oblicuos decorativos se encuentran exclusivamente en las caras laterales y aparecen en cuatro piezas.

En las dos piezas cuyo fuste es curvo (K9.2.4 y K9.2.13) los trazos decorativos son simples y aparecen junto a trazos funcionales. 


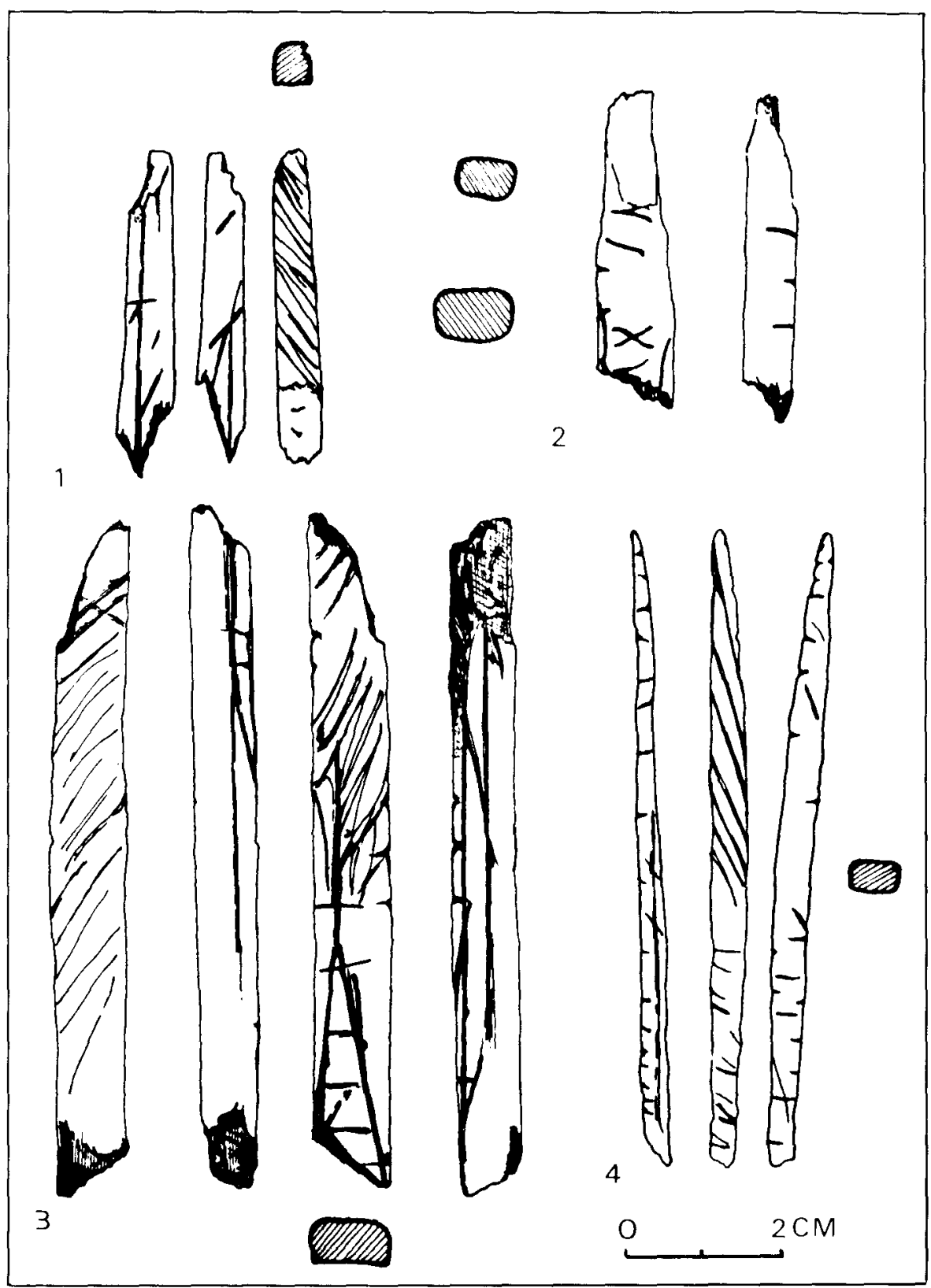

Fig. 2. Industria ósea de Altamira 1981. 1) K9.2.9; 2) K8.2.10; 3) L9.2.14 y 4) K9.2.5 
REFLEXIONES FINALES

La observación de esta serie de piezas inéditas de industria ósea contribuye a reafirmar un criterio ya establecido en los estudios sobre la Cornisa Cantábrica: la movilidad territorial de los grupos del Magdaleniense Inferior.

Si nos remitimos a las decoraciones de las piezas, los "tectiformes" nos acercan a Castillo y a Juyo, 10 que podria implicar movimientos territoriales que siguiendo los valles perpendiculares y transversales unirian las agrupaciones 5 y 6 establecidas por P. Utrilla (1981:261).

En esta serie, dos yacimientos considerados como base de hábitat (Altamira y Castillo) se unen a una estación con especialización en la caza de una determinada especie. En este sentido conviene señalar que si bien las referencias de Castillo proceden de una clasificación antigua, el desglose de la fauna implica una caracterización parecida (expresada en número minimo de individuos: ciervo 167, Bos sp. 10, rebeco 9 y équidos 9, con presencia de otras especies). La fauna de Altamira aún está por revisar, pero en una apreciación simple se muestran muy numerosos los restos de ciervo. En cuanto a la ocupación. Juyo se muestra también en las actuales excavaciones como yacimiento complejo.

Si nos remitimos a otras caracteristicas, en el recuento de la industria lítica (exc. 1981) de Altamira, J. González Echegaray establece una correlación. Ilamada facies Altamira, con los niveles 8 y 7 de Castillo. El nivel 8 de Juyo tiende a aproximarse al mismo. En este nivel es donde comienzan a aparecer los omóplatos grabados.

En la actualidad muchos autores abogan por la asociación de los omóplatos de Altamira al Magdaleniense Inferior (FREEMAN, 1988; UTRILLA, 1989:406). Cuando estudiamos Castillo, siempre fuimos proclives a considerar los de Altamira como Magdaleniense Inferior (CABRERA, 1984:410); posición también defendida por Obermaier y Breuil. Con el tiempo hemos ido afirmándonos en esta idea, considerando otros factores (CABRERA y Giménez, 1989) como son la presencia de "máscaras" en el arte parietal (Castillo y Altamira) y el reflejo de la industria ósea. A ello se uniría la similitud de factura de bisontes polícromos ya sugerida por Alcalde del Río, Breuil y Sierra (1912) y puesta de relieve en cuanto a la técnica, realización y concepción del artista entre Altamira y Castillo (MúzQuIz, 1990). 
AGRADECIMIENTOS

Agradecemos a Joaquín González Echegaray el habernos invitado a participar en el estudio de los materiales de la campaña de 1981 en la que tan amablemente nos acogió.

Nos gustaría mencionar el asesoramiento informático que nos brindó Federico Bernaldo de Quirós y a todos nuestros compañeros del CIMA.

No podriamos terminar sin mostrar nuestro reconocimiento a Gloria Pino Fernández, cuyos dibujos ilustran este artículo; y a A. Fleming por su inestimable apoyo. 


\section{BIBLIOGRAFIA}

Alcalde del Rio, H.; Breull, H. y Sierra, L., 1912: Les cavernes de la Region Cantabrique. Mónaco.

Barandiaran Maestu, I., 1967: “El Paleomesolítico del Pirineo Occidental. Bases para una sistematización tipológica del instrumental óseo paleotítico". Monografías Arqueológicas, III. Zaragoza.

- 1985: Industria ósea paleolítica de la cueva de El Juyo, excavaciones de 1978 y 1979. "Excavaciones en la cueva de El Juyo", Monografias del CIMA núm. 14, págs. 161-194.

Cabrera Valdes, V., 1984: El yacimiento de la cueva de El Castillo. Biblioteca Praehistorica Hispana XXI. Madrid.

Cabrera Valdes, V. y Giménez la Rosa, M., 1989: "Arte mueble paleolítico en la Cornisa Cantábrica", Revista de Arqueología núm. 103, págs. 12-24.

CONKEY, M., 1987: “L'art mobilier et l'établissement de géographies sociales", Actas del Colloque International d'Art Mobilier Paleolithique, Foix, págs. 293-295.

CORCHÓN RODRIGUEZ, S., 1986: “Arte mueble paleolítico cantábrico: contexto y análisis interno", Monografias del CIMA núm. 16.

Delporte, H.; Hahn, J.; Mons, L.; Picon, G. et Sonneville-Bordes, D., 1988: Fiches typologiques de l'industrie osseuse préhistorique. Cahier I. Sagaies. UISPP Université de Provence.

Freeman, L. G., 1988: "The stratigraphic sequence at Altamira, 18801981 ", Espacio. Tiempo y Forma. Serie I. Prehistoria y Arqueología, t. I, págs. $149-163$.

GonZALEZ EChegaray, J., 1988: “El magdaleniense de Altamira", Espacio, Tiempo y Forma, Serie I, Prehistoria y Arqueologia, t. I, págs. 165-171.

Múzuiz, M., 1990: "El pintor de Altamira pintó en la cueva de El Castillo", Revista de Arqueología, num. 114; págs. 14-22.

Utrilla Miranda, P., 1981: «El Magdaleniense inferior y medio en la costa cantábrica", Monografías del CIMA, núm. 4.

- 1987a: “Bases objetivas de la chronologie de l'art mobilier sur la Côte Cantabrique", Actas del Colloque International d'Art Mobilier Paleolithique, Foix; págs. 83-104.

- 1987b: «El Magdaleniense Inferior de la Costa Cantábrica», Actas del Colloque de Mayence Le Magdalenien en Europe. la structuration du Magdalenien. Etudes et Recherches Arquéologiques de I'Universite de Liège, núm. 38, págs. 399-418. 